\title{
Optional shift behavior of albino rats
}

\author{
Tracy S. Kendler, BARNARD COLLEGE; Howard H. Kendler, UNIVERITY OF CALFORNLA, \\ SANTA BARBARA; and Carole K. Silfen, MCMASTER UNIVERITY
}

\begin{abstract}
When children are presented with an optional shift after learning a discrimination to criterion the proportion of reversal shifts increases and nonselective shifts decrease with age. The present research shows that when albino rats are presented with a similar option the proportion of reversal shifts is practically zero. They make either a nonselective or an extradimensional shift with the former more likely than the latter. A continuity between the behavior of rats and very young children is suggested.
\end{abstract}

\section{Problem}

Infrahuman organisms usually require more trials to make a reversal shift (from $\mathrm{S}+$ to $\mathrm{S}-$ ) than an extradimensional shift (from $\mathrm{S}+$ to a stimulus on a previously irrelevant dimension)! On the other hand, adult human Ss learn a reversal more easily than an extradimensional shift (Kendler and Kendler, 1962). Kendler, Kendler and Learnard (1962) assumed that this species difference is due to the tendency for adult humans to make implicit responses which mediate their overt behavior. In order to investigate the development of mediating responses among children, they compared the relative ease of learning two kinds of shifts. Each $\mathrm{S}$ had the option of learning a second discrimination by executing either a reversal of the first, or an extradimensional shift, or by responding nonselectively. The results, based on stimuli differing in size and brightness, are seen in Fig. 2. The present research continues the investigation of continuities and discontinuities between human and infrahuman behavior by applying the optional shift procedure to rats.

\section{Method}

Twenty-four male albino rats between 90 and 120 days old were trained to discriminate between two squares that differed simultaneously in brightness and size (Fig. 1) on an apparatus patterned after Gibson and Walk (1956). The large squares measured $7.0 \mathrm{~cm}$, and the small squares $3.8 \mathrm{~cm}$ on a side. Each square, which was painted either flat black or flat white, had a $3.2 \mathrm{x}$ $2.8 \mathrm{~cm}$ hinged door cut in its center. Behind the door was a small metal food tray which always contained a

Extradimensional shifts have often been referred to as nonreversal shifts.

2 Nonselective shifts were referred to as inconsistent shifts by Kendler, Kendler, and Learnard. food pellet.

Each $\mathrm{S}$ was pretrained for three days during which it became accustomed to the apparatus and learned to eat from the food trays. In the initial discrimination, $\mathrm{S}$ was presented alternately with two pair of stimuli that differed simultaneously on both dimensions. For any given S, only one stimulus value was correct and only one dimension was relevant. Each stimulus value, black, white, large, and small, was $\mathrm{S}+$ for six Ss. A correct response consisted of nosing open the unlatched door of S+toget a pellet; an incorrect response, of nosing $\mathrm{S}-$, which was latched from behind. After each response, correct or incorrect, guillotine doors in front of both stimuli were lowered and $S$ returned to the starting box. After $S$ made a correct response, the stimuli were changed for the subsequent trial according to a prearranged counterbalanced order. Twenty daily trials were given during training until a criterion of 18 of them, including 9 out of the last 10 , were correct.

The day after criterion was attained a second discrimination, the optional shift, was presented. This utilized only one of the two pair of stimuli, and on this pair the reinforcement pattern was reversed. Ss received 20 daily trials and were run to the same criterion as the initial discrimination. Since both dimensions were relevant, Shad the option of learning by responding to the previous S-, e. g. white, or to the stimulus of the previously irrelevant dimension, e. $g$. small, or to both stimulus values, e. g. small-white.

The day after criterion was attained on the optional shift, the test series was presented. The pair that had not been presented during the optional shift was the test pair. This was presented 10 times, and response to either member was rewarded. If $S$ responded 8 or more times to the previous S-, e.g. large white, its shift was classified as reversal. If it responded 8 or more times to the previously irrelevant stimulus, e.g. small black, its shift was classified as extradimensional. If $S$ made less than 8 out of the 10 responses to either member of the test pair its shift was classified as nonselective $^{2}$. The other stimulus pair, which retained the

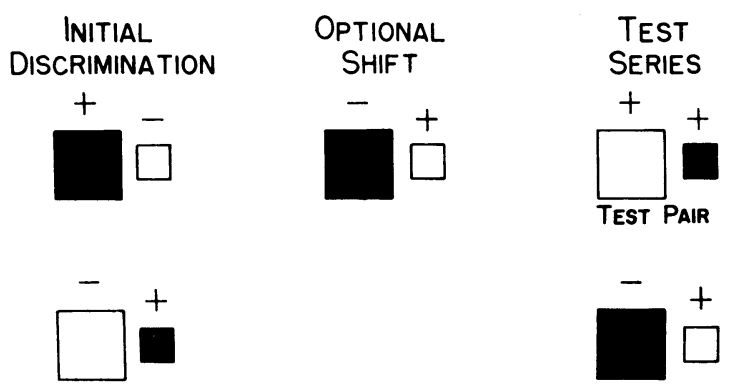

Fig. 1 An illustration of the stimuli and one of the reinforcement patterns used to investigate the optional shift behavior of albino rats. 
same reinforcement pattern as in the optional shift, was presented along with the test pair in the same alternating order as in the initial discrimination in order to keep $\mathrm{S}$ responding during the test trials on the same basis as it had during the optional shift.

This procedure is comparable in several respects to the one used with the children: in stimulus dimensions, in size and brightness relationships, and in the optional shift technique. The apparatus used was different, and so were several details of the training including the fact that the children completed the experiment in one sitting and were reinforced by marbles instead of food.

\section{Results and discussion}

The mean number of trials required to reach criterion was 180.8 for the initial discrimination and 111.7 for the optimal shift. The corresponding standard deviations were 76.0 and 46.5. The optimal shift results were interperted by assuming that if the rats responded nonselectively during the test series then the choice of either of the two members of the test pair would be equally likely and the choice behavior could be expected to approximate the binomial expansion $(.5 \mathrm{x}+$ $.5 y)^{10}$. The theoretical probabilities converted to percentages are as follows: reversal shifts = $5.5 \%$, nonselective shifts $=89.0 \%$, and extradimensional shifts $=5.5 \%$. The corresponding obtained percentages were: reversal shifts $=4.2 \%$, nonselective shifts $=62.5 \%$, and extradimensional shifts $=33.3 \%$. Hence, the percent of reversal shifts was consonant with the nonselective hypothesis. But the percent of nonselective shifts was less and the percent of extradimensional shifts was greater than the hypothesis predicts. Put another way, if there is a departure from the nonselective hypothesis it is toward extradimensional shifts, not reversal shifts.

The rats' shift behavior, compared with the children's, presents an interesting continuity (Fig. 2). This is consonant with a pretheoretical model (Kendler and Kendler, 1962) which proposes that the species difference in shift behavior is due to the tendency for relatively mature humans, in contrast to young children and infrahuman animals, to make covert representational responses which mediate their overt behavior. With increasing $\mathrm{CA}$ the probability of mediating responses increases and, therefore, so does the

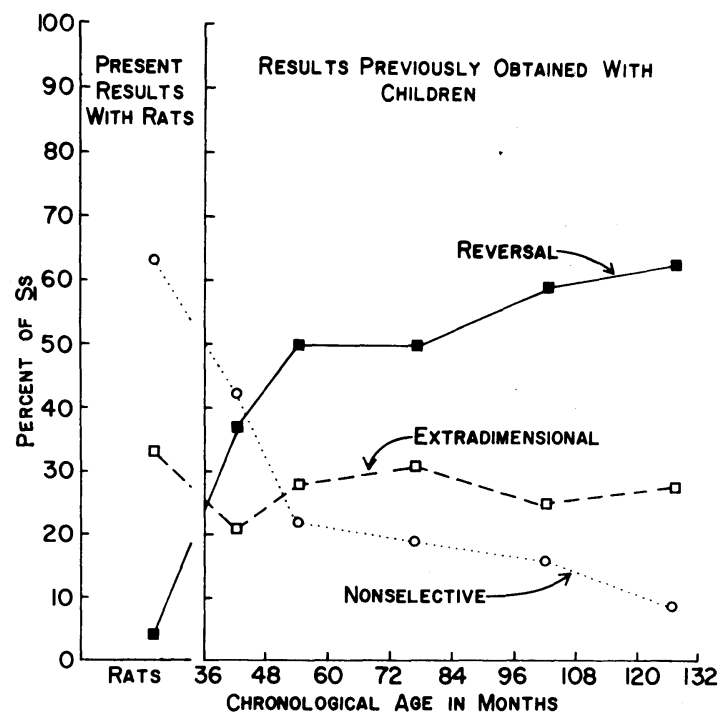

Fig. 2 The percent of Ss responding in each optional shift category for 90-120 day old rats and children of different ages.

difference between human and infrahuman shift performance. The model is not precise enough to locate where on the lower developmental continuum the rat results should be placed. The point depicted in Fig. 2 which happens to correspond with a CA of 24 months was determined by a visual goodness of fit. It remains to be seen whether the shift behavior of two year old children corresponds with that of the 2 to 3 month old rats.

\section{References}

GIBSON, E. J. and WALK, R. D. The effect of prolonged exposure to visually presented patterns on learning to discriminate them.J. comp. physiol. Psychol., 1956, 49, 239-242.

KENDLER, H. H. and KENDLER, T. S. Vertical and horizontal processes in problem solving. Psychol. Rev., 1962, 69, 1-16.

KENDLER, T. S., KENDLER, H. H., and LEARNARD, B. Mediated responses to size and brightness as a function of age. Amer. J. Psychol., 1962, 75, 571-586.

\section{Acknowledgment}

This research was supported by a grant from the National Science Foundation. 